\title{
Orbital angular momentum of partially coherent beams
}

\author{
J. Serna and J. M. Movilla \\ Departamento de Óptica, Facultad de Ciencias Físicas, Universidad Complutense de Madrid, 28040-Madrid, Spain
}

Received July 10, 2000

\begin{abstract}
The definition of the orbital angular momentum established for coherent beams is extended to partially coherent beams, expressed in terms of two elements of the beam matrix. This extension is justified by use of the Mercer expansion of partially coherent fields. General Gauss-Schell-model fields are considered, and the relation between the twist parameter and the orbital angular momentum is analyzed. (C) 2001 Optical Society of America

OCIS code: 030.1640 .
\end{abstract}

Over the past ten years there has been intensive investigation of the angular momentum of electromagnetic fields. ${ }^{1-9}$ In the paraxial approach $^{2}$ the angular momentum of a light beam can be decomposed into two terms, one proportional to the amount of circular polarization, the spin term, and the other, the so-called orbital angular momentum, related to the spatial structure of the phase. However, most studies of orbital angular momentum have analyzed only completely coherent beams, leaving aside the more general case of partially coherent fields. Recently a treatment of partially coherent beams with orbital angular momentum was introduced, but it was restricted to beams that can be constructed from a superposition of Laguerre-Gauss modes with the same azimuthal number $l .^{6}$

In this Letter we propose an extension of the existing treatment of the orbital angular momentum, which is valid for partially coherent beams and is based on the well-known Wigner distribution function. ${ }^{10}$ From such a definition, which can be used to clarify several experimental situations, ${ }^{8,9}$ a combination of two elements of the so-called beam matrix will take a clear physical interpretation. This definition is rigorously justified by use of the Mercer expansion of the cross-spectral density function of partially coherent beams. ${ }^{11}$ Finally, this definition is applied to general Gaussian-Schell-model (GSM) beams. ${ }^{12,13}$ For these beams the relation between the twist parameter and the orbital angular momentum is established.

Let us start by introducing $\mathbf{L}$, the angular momentum density per unit length along the $z$ axis of a light beam. ${ }^{1}$ In free space

$$
\mathbf{L}=\iint \mathbf{M}(\mathbf{r}) \mathrm{d} x \mathrm{~d} y,
$$

where $\mathbf{r}$ is the position vector and $\mathbf{M}(\mathbf{r})=\mathbf{r} \times \mathbf{S} / c^{2}$ is the angular momentum density of the light beam expressed in terms of the Poynting vector, $\mathbf{S}=\epsilon_{0} c^{2} \mathbf{E} \times$ $\mathbf{B}$, where $\epsilon_{0}$ is the vacuum permittivity and $c$ is the speed of light.

We are interested in the angular momentum density per unit length of a monochromatic uniformly polarized beam propagating along the $z$ direction in the paraxial approach. We can obtain a convenient representation of such an optical beam in the Lorentz gauge by choosing the following vector potential:

$$
\mathbf{A}(\mathbf{r})=A_{0}(\mathbf{r}) \exp [-i(\omega t-k z)] \mathbf{e},
$$

where $t$ is the time variable, $\omega$ is the angular frequency, $k=\omega / c$, and $\mathbf{e}=\left(e_{x}, e_{y}, 0\right)$ is a unit vector that contains the polarization information. Considering time-averaged quantities, we can write the Poynting vector in vacuum as

$$
\overline{\mathbf{S}}=\left(\epsilon_{0} c^{2} / 2\right) \operatorname{Re}\left[\mathbf{E}(\mathbf{r}) \times \mathbf{B}^{*}(\mathbf{r})\right],
$$

where the overbar symbolizes the time average.

Within the Lorentz gauge the field vectors $\mathbf{E}$ and $\mathbf{B}$ can be derived from the vector potential. Neglecting the second derivatives of $A_{0}(\mathbf{r})$ and the first derivatives with respect to longitudinal variable $z$, and using definitions (1) and (3), we can obtain the time-averaged linear density of the angular momentum $\overline{\mathbf{L}}$ for the vector potential proposed in Eq. (2). At a certain plane $z$ its components are

$$
\begin{aligned}
& \bar{L}_{x}=I(\langle y\rangle-z\langle v\rangle) / c^{2}, \\
& \bar{L}_{y}=I(-\langle x\rangle+z\langle u\rangle) / c^{2}, \\
& \bar{L}_{z}=I(\langle x v\rangle-\langle y u\rangle) / c^{2}+s_{3} / c \omega,
\end{aligned}
$$

where $s_{3}=2 I \operatorname{Im}\left(\overline{e_{x} e_{y}{ }^{*}}\right)$ is the fourth Stokes parameter of the beam, $I$ is the power that can be calculated from the $z$ component of $\overline{\mathbf{S}}$ as

$$
I=\iint \bar{S}_{z} \mathrm{~d} x \mathrm{~d} y=\iint|\psi|^{2} \mathrm{~d} x \mathrm{~d} y
$$

and $\psi(\mathbf{r}),\langle x\rangle,\langle u\rangle,\langle x y\rangle$, and $\langle y u\rangle$ are defined as

$$
\begin{aligned}
\psi(\mathbf{r}) & =i \omega\left(\sqrt{\epsilon_{0} c} / 2\right) A_{0}(\mathbf{r}) \\
\langle x\rangle & =\frac{1}{I} \iint x|\psi|^{2} \mathrm{~d} x \mathrm{~d} y \\
\langle u\rangle & =\frac{i}{2 k I} \iint\left(\psi \frac{\partial \psi^{*}}{\partial x}-\psi^{*} \frac{\partial \psi}{\partial x}\right) \mathrm{d} x \mathrm{~d} y,
\end{aligned}
$$




$$
\begin{aligned}
& \langle x v\rangle=\frac{i}{2 k I} \iint x\left(\psi \frac{\partial \psi^{*}}{\partial x}-\psi^{*} \frac{\partial \psi}{\partial y}\right) \mathrm{d} x \mathrm{~d} y, \\
& \langle y u\rangle=\frac{i}{2 k I} \iint y\left(\psi \frac{\partial \psi^{*}}{\partial x}-\psi^{*} \frac{\partial \psi}{\partial x}\right) \mathrm{d} x \mathrm{~d} y .
\end{aligned}
$$

Definitions for $\langle y\rangle$ and $\langle v\rangle$ are symmetric to those given for $\langle x\rangle$ and $\langle u\rangle$, respectively.

In the previous expressions $\langle x\rangle,\langle y\rangle,\langle u\rangle$, and $\langle v\rangle$ are the so-called first-order irradiance moments of the beam. ${ }^{10}$ They represent the beam center of gravity $(\langle x\rangle,\langle y\rangle)$ and the mean direction of the beam propagation $(\langle u\rangle,\langle v\rangle)$. With that in mind, Eqs. (4) and (5) come as no surprise. When we define the angular momentum with respect to the origin, a beam that does not pass through $x=y=0$ or form an angle with the $z$ direction will introduce angular momentum along the transversal directions. Therefore, suitable translation and rotation of the Cartesian coordinate system, such that $\langle x\rangle=\langle y\rangle=\langle u\rangle=\langle v\rangle=0$, leaves only the nontrivial longitudinal angular momentum component, $\bar{L}_{z}$. In that case the time-averaged angular momentum flux of a coherent beam through a $z$ plane, $\bar{J}_{z}=\bar{L}_{z} c$, is given $\mathrm{by}^{7}$

$$
\bar{J}_{z}=\left(\frac{I}{c}\right)(\langle x v\rangle-\langle y u\rangle)+\frac{s_{3}}{\omega} .
$$

As expected, the longitudinal component of the angular momentum flux is composed of two terms. The polarization, or spin, term,

$$
\bar{J}_{z} S=\frac{s_{3}}{\omega},
$$

depends on the Stokes parameter $s_{3}$ and therefore is proportional to the amount of circular polarization of the beam. The spatial term,

$$
\bar{J}_{z}{ }^{L}=\left(\frac{I}{c}\right)(\langle x v\rangle-\langle y u\rangle),
$$

is expressed here as the difference of two second-order beam moments that, for coherent fields, are related to the phase spatial structure. Equation (15) gives the correct result for Laguerre-Gauss beams, and it is also valid for other types of coherent beam, such as those considered in Ref. 4. From now on we shall focus our attention on the orbital angular momentum term.

At this point it is interesting to consider the second-order moments characterization of beams. Such characterization relies on the so-called beam matrix. $^{10,12}$ This matrix is a real $4 \times 4$ symmetric positive-definite matrix with only ten independent elements. All the elements are related to spatial characteristics of the beam, such as beam widths, divergences, and radii of curvature. Only $\langle x v\rangle-\langle y u\rangle$, the antisymmetric part of one of the submatrices of the beam matrix, remains with no clear physical interpretation. Now, from Eq. (15), it can be concluded that this term is proportional to the orbital angular momentum transported by the beam. Also note that, as expected, $\bar{J}_{z}$ is invariant under rotation and in free-space propagation.
Although Eq. (15) was derived only for totally coherent fields, we could think about using it for general partially coherent beams with the same physical meaning. For such beams the beam matrix is also defined with the same interpretation of nine of its elements, those related to beam widths, divergences, and generalized radii of curvature. In this sense it seems plausible to consider Eq. (15) the orbital angular momentum transported by general partially coherent fields. In that case, the second-order moments should be defined in terms of the Wigner distribution function $h(x, y, u, v)$ of the beam, ${ }^{10}$ namely,

$$
\begin{aligned}
& \langle x v\rangle=\left(\frac{1}{I}\right) \iiint \int x v h(x, y, u, v) \mathrm{d} x \mathrm{~d} y \mathrm{~d} u \mathrm{~d} v, \\
& \langle y u\rangle=\left(\frac{1}{I}\right) \iiint \int y u h(x, y, u, v) \mathrm{d} x \mathrm{~d} y \mathrm{~d} u \mathrm{~d} v,
\end{aligned}
$$

where $u$ and $v$ represent angles of propagation (the evanescent waves are not taken into account). It is important to note that Eqs. (11) and (12) are equivalent to Eqs. (16) and (17) in the case of totally coherent beams.

The previous plausibility argument can be rigorously justified by means of the so-called Mercer expansion of partially coherent beams. According to Ref. 11, the cross-spectral density function $\Gamma\left(\mathbf{r}_{1}, \mathbf{r}_{2}\right)$ of such fields, which contains the same information as $h(x, y, u, v){ }^{10}$ can be expressed as an incoherent superposition of cross-spectral density functions associated with coherent fields:

$$
\Gamma\left(\mathbf{r}_{1}, \mathbf{r}_{2}\right)=\sum_{n} \Gamma_{n}\left(\mathbf{r}_{1}, \mathbf{r}_{2}\right)
$$

where

$$
\Gamma_{n}\left(\mathbf{r}_{1}, \mathbf{r}_{2}\right)=\psi_{n}^{*}\left(\mathbf{r}_{1}\right) \psi_{n}\left(\mathbf{r}_{2}\right)
$$

corresponds to totally coherent beams. In this expansion $\psi_{n}\left(\mathbf{r}_{2}\right)$ are solutions of the following integral equation:

$$
\iint \Gamma\left(\mathbf{r}_{1}, \mathbf{r}_{2}\right) \psi_{n}\left(\mathbf{r}_{1}\right) \mathrm{d} x \mathrm{~d} y=I_{n} \psi_{n}\left(\mathbf{r}_{2}\right),
$$

where the eigenvalue $I_{n}$ is the power content associated with $\psi_{n}$ and the eigenfunctions are orthogonal:

$$
\iint \psi_{n}^{*}(\mathbf{r}) \psi_{m}(\mathbf{r}) \mathrm{d} x \mathrm{~d} y=I_{n} \delta_{n, m}
$$

It is interesting to note that, because the Wigner distribution is the Fourier transform of the cross-spectral density function and the Fourier transform is linear, Eq. (18) can be rewritten in terms of $h(x, y, u, v)$.

We can assign an orbital angular momentum flux $\bar{J}_{z}^{(n)}$ to each coherent mode $n$ :

$$
\bar{J}_{z}^{(n)}=\left(I_{n} / c\right)\left[\langle x v\rangle_{n}-\langle y u\rangle_{n}\right]
$$

Inasmuch as the total cross-spectral density function $\Gamma$ is a superposition of mutually incoherent terms $\Gamma^{(n)}$ 
we can sum all the contributions $\overline{\boldsymbol{J}}_{z}^{(n)}$ to obtain the total orbital angular momentum:

$$
\bar{J}_{z}=\frac{1}{c} \sum_{n} I_{n}\left(\langle x v\rangle_{n}-\langle y u\rangle_{n}\right) .
$$

However, by virtue of the Mercer expansion [written in terms of $h(x, y, u, v)]$, the second-order moments $\langle x v\rangle$ and $\langle y u\rangle$ of a partially coherent field can be expressed in terms of the same second-order moments associated with each mode $n$ as

$$
\begin{aligned}
\langle x v\rangle & =(1 / I) \sum_{n} I_{n}\langle x v\rangle_{n}, \\
\langle y u\rangle & =(1 / I) \sum_{n} I_{n}\langle y u\rangle_{n},
\end{aligned}
$$

where $I=\sum_{n} I_{n}$ is the power of the partially coherent beam. Substituting Eqs. (24) and (25) into Eq. (23), we arrive at

$$
\bar{J}_{z}=\left(\frac{I}{c}\right)(\langle x v\rangle-\langle y u\rangle),
$$

in complete agreement with Eq. (15).

As a particular case let us consider GSM beams. ${ }^{12,13}$ These are the most general beams with Gaussian profiles in irradiance and in transversal spatial coherence, and they are described in terms of three $2 \times 2$ symmetric matrices, $\boldsymbol{\sigma}_{I}$ (beam width), $\boldsymbol{\sigma}_{g}$ (transverse coherence width), $\mathbf{R}$ (curvature radius), and a scalar $\tau$ (the twisted phase parameter). Following Eq. (15) and using Eq. (20) of Ref. 12 (with the correct sign), we find that the orbital angular momentum of GSM fields is given by

$$
\bar{J}_{z}=\left(\frac{I}{2 c}\right)\left\{\operatorname{Tr}\left[\left(\mathbf{R}^{-1} \boldsymbol{\sigma}_{I}^{2}-\boldsymbol{\sigma}_{I}^{2} \mathbf{R}^{-1}\right) J\right]-2 \tau \operatorname{Tr}\left(\boldsymbol{\sigma}_{I}^{2}\right)\right\},
$$

where

$$
\mathbf{J}=\left[\begin{array}{cc}
0 & 1 \\
-1 & 0
\end{array}\right]
$$

A first conclusion is that $\bar{J}_{z}$ is independent of the coherence properties of the beam given by $\boldsymbol{\sigma}_{g}$ (although $\tau$ is limited by $\boldsymbol{\sigma}_{g}$, it does not depend on $\left.i t^{12}\right)$. We can also see that there are two contributions to $\overline{\mathbf{J}}_{z}$. The first term depends on $\boldsymbol{\sigma}_{I}$, and $\mathbf{R}$ and is zero only when both matrices have the same orientation. The second term vanishes only when $\tau=0$. Therefore $\tau$ cannot be identified, in general, with the orbital angular momentum (also note that in general $\tau$ is not even invariant in free propagation). In this sense we can find beams that lend both contributions to the angular momentum (such as the pseudoaligned simple astigmatic beams ${ }^{12}$ ), beams that fulfill $\bar{J}_{z} \neq 0$ but with $\tau=0$ (for example, coherent Gaussian beams whose intensity profiles rotate in free propagation ${ }^{5}$ ) or beams (for example, the family of twisted GSM beams ${ }^{14}$ ) for which twist and orbital angular momentum are equivalent because $\boldsymbol{\sigma}_{I}$ and $\mathbf{R}$ have the same orientation. Finally, inasmuch as each term could be positive or negative, there are beams for which the $\boldsymbol{\sigma}_{I}-\mathbf{R}$ contribution and the $\tau$ contribution compensate for each other, giving a beam that does not transport orbital angular momentum.

We thank G. Piquero and G. Nemeş for helpful discussions. This study was supported by the Comision Interministerial de Ciencia y Tecnología of Spain under project PB97-0295. J. Serna's e-mail address is fiopt01@sis.ucm.es.

\section{References}

1. L. Allen, M. W. Beijersbergen, R. J. C. Spreeuw, and J. P. Woerdman, Phys. Rev. A 45, 8185 (1992).

2. S. M. Barnett and L. Allen, Opt. Commun. 110, 670 (1994).

3. H. He, M. E. J. Friese, N. R. Heckenberg, and H. Rubinsztein-Dunlop, Phys. Rev. Lett. 75, 826 (1995).

4. N. B. Simpson, K. Dholakia, L. Allen, and M. J. Padgett, Opt. Lett. 22, 52 (1997).

5. J. Courtial, K. Dholakia, L. Allen, and M. J. Padgett, Opt. Commun. 144, 210 (1997).

6. G. S. Agarwal, J. Opt. Soc. Am. A 16, 2914 (1999).

7. A. T. Friberg, C. Gao, B. Eppich, and H. Weber, Proc. SPIE 3110, 317 (1997).

8. J. Arlt, K. Dholakia, L. Allen, and M. J. Padgett, Phys. Rev. A 59, 3950 (1999).

9. J. Serna, F. Encinas-Sanz, and G. Nemeş, in Proceedings of the 5th International Workshop on Laser Beam and Optics Characterization, H. Weber and H. Laabs, eds. (Technische Universität-Optics Institut, Berlin, 2000).

10. M. J. Bastiaans, J. Opt. Soc. Am. 69, 1710 (1979).

11. E. Wolf, J. Opt. Soc. Am. 72, 343 (1982).

12. G. Nemeş and J. Serna, in 4th International Workshop on Laser Beam and Optics Characterization, A. Giesen and M. Morin, eds., (VDI-Technologiezentrum, Dusseldorf, Germany, 1997), pp. 92-105.

13. R. Simon, E. C. G. Sudarshan, and N. Mukunda, Phys. Rev. A 29, 2419 (1985).

14. R. Simon and N. Mukunda, J. Opt. Soc. Am. A 10, 95 (1993). 